\title{
HIV Infection Alters the Spectrum of HPV Subtypes Found in Cervical Smears and Carcinomas from Kenyan Women
}

\author{
Innocent O. Maranga ${ }^{1,2}$, Lynne Hampson ${ }^{1}$, Anthony W. Oliver ${ }^{1}$, Xiaotong $\mathrm{He}^{1}$, Peter Gichangi ${ }^{2}$, \\ Farzana Rana ${ }^{3}$, Anselmy Opiyo ${ }^{4}$ and Ian N. Hampson ${ }^{*}, 1$ \\ ${ }^{I}$ Viral Oncology Laboratories, University of Manchester Institute of Cancer Sciences, Research Floor 5, St Mary's \\ Hospital, Manchester, M13 9WL, UK \\ ${ }^{2}$ Departments of Obstetrics and Gynaecology, University of Nairobi, Nairobi, Kenya \\ ${ }^{3}$ Aga Khan University Hospital, Nairobi, Kenya \\ ${ }^{4}$ Cancer Treatment Centre, Kenyatta National Hospital, Kenya
}

\begin{abstract}
Infection with high risk HPV is implicated in pre-cancerous squamous intraepithelial lesions and their progression to cervical cancer. In the developed countries, infection with HPV 16 and 18 accounts for $\sim 70 \%$ of cervical cancers, but it has been established that HPV type prevalence differs according to worldwide geographical location. In sub Saharan Africa infection with HPV is known to be augmented by HIV, which is endemic in this region. It is not yet clear, however, whether this ultimately influences progression to cervical cancer. Papillocheck ${ }^{\mathrm{TM}}$ and multiplex PCR were used to determine the range of HPV genotypes found in cervical smears and carcinomas from HIV positive and negative Kenyan women. Smear samples from HIV-positive women had a higher prevalence of: multiple HPV infections; high-risk HPVs 52, 58, 68, potential high risk 53/70, low-risk $44 / 55$ and abnormal cytology compared to HIV-negative women. A low overall prevalence $(\sim 8 \%)$ of types $16 / 18$ was found in all smear samples tested $(n=224)$ although this increased in invasive cervical carcinoma tissues to $\sim 80 \%$ for HIV-negative and $\sim 46 \%$ for HIV-positive women. Furthermore, HPV45 was more common in cervical carcinoma tissues from HIV-positive women.

In summary HIV infection appears to alter the spectrum of HPV types found in both cervical smears and invasive cervical carcinomas. It is hypothesised there could be a complex interplay between these viruses which could either positively or negatively influence the rate of progression to cervical cancer.
\end{abstract}

Keywords: Africa, cervical smear, HIV, HPV, Invasive cervical cancer, Subtype.

\section{INTRODUCTION}

Infection with oncogenic HPV has now been firmly established as the aetiological agent for invasive cervical cancer (ICC) [1-3]. Globally there are 270,000 deaths from this disease per annum with over $85 \%$ of these occurring in low resource countries [4]. For instance, in Kenya it is the most common cancer per se accounting for between $18-23 \%$ of all diagnosed cancer cases [5]. It is also considered an AIDS defining diagnosis by the CDC [6] and in 2007 the World Health Organisation added ICC to the stage 4 HIV/AIDS classification of its clinical staging and case definition of HIV for resource-constrained settings [7]

Both HPV and HIV are sexually transmitted and infection with either is known to facilitate infection with the other [8-10]. For this reason the possible relationship between the two viral infections and cervical cancer has been the focus of numerous studies [11-13]. Globally, an

*Address correspondence to this author at the Viral Oncology Laboratories, University of Manchester Institute of Cancer Sciences, Research Floor 5, St Mary's Hospital, Manchester, M13 9WL, UK; Tel: +441617016938;

E-mail: ian.hampson@manchester.ac.uk increased risk of HPV infection and cervical pre-cancerous squamous intraepithelial lesions (SIL's) has been found in HIV-positive women [14, 15]. In some European populations a positive association of HIV infection and ICC has also been documented [16-18] but the picture is less clear in Africa where evidence is conflicting [11-13, 19-23]. For example, recent work carried out in Cote d'Ivoire supported the hypothesis that HIV infection could act as a co-factor for ICC in HPV positive women [19] whilst a lack of association between HIV and ICC was reported in Ugandan women [24].

The incongruent findings are cause for concern especially as Kenya currently has 1.2 million HIV-positive women who may well have an increased risk of developing ICC $[20,21]$. Furthermore there is evidence that the vaccination program against high-risk HPV types 16 and 18, which has been successfully employed in many developed countries, may not be as effective in HIV positive individuals. This is as HIV positive women have been shown to have much more diverse HPV types in their cervical tissues [25, 26]. Surprisingly, despite the fact that sub-Saharan Africa has the highest incidence of HIV and ICC, the HPV type distribution has not been well defined in this region [1]. Clearly, it 
follows that the acquisition of baseline data on HPV prevalence and genotype distribution with respect to HIV status will be important to enable evaluation of the possible impact of prophylactic HPV vaccines in this area of the world, particularly as it contains two thirds of the worlds HIV burden [27, 28]. In addition, further elucidation of the relationship between HIV and different HPV types in cervical cancer must be sought. Therefore, we now report analysis of the HPV genotypes found in cervical smears and ICC's from HIV-positive and HIV-negative Kenyan women with a view to clarifying the relationship between these viruses and ICC in this study population.

\section{MATERIALS AND METHODS}

\section{Cervical Smear Study Population and Setting}

A case control study was designed and conducted at Kenyatta National Hospital (KNH), Nairobi, Kenya, which is the country's main referral hospital. Specimen analysis was done at the Viral Oncology Laboratories, University of Manchester, UK. Consecutive female patients attending Specialist HIV Clinic and Family Planning Clinic were recruited following written informed consent between April 2008 and February 2009. Women of mainly reproductive age were included (18-50 years), while those who had prior ablative procedures for cervical disease and hysterectomies were excluded. A total of 224 patients were recruited; 111 were HIV-negative while 113 were HIV-positive (half of these were on Highly Active Antiretroviral Therapy, HAART). After undergoing voluntary counselling and testing for HIV, a structured questionnaire was administered (asking socio-demographics and sexual histories) and a blood sample taken. A HIV test was done on all the 224 patients using the Determine ${ }^{\circledR}$ test kit (Abbot Pharmaceuticals, Chicago, USA), and if positive, was confirmed by Uni-Gold ${ }^{\mathbb{B}}$ (Trinity Biotech Plc, Ireland). All HIV positive patients had CD4 counts undertaken and these ranged between $72-940 / \mathrm{mm}^{3}$ with a mean of $393 / \mathrm{mm}^{3}$.

\section{Ethics Statement}

Ethical approval was granted by both the Kenyatta National Hospital Ethics Committee (05.12.2007: KNHERC/01/4988) and the Oldham Ethics Committee UK (26.01.2009: amendment 5 project 07/Q1405/14).

\section{Liquid Based Cytology}

All patients were examined with a speculum and cervical samples collected using a Cervex brush which was stirred into a vial of PreservCyt transport solution (ThinPrep Pap Test, Hologic Inc, USA). Cytospins thereof were stained with Papanicolaou (pap) stain and were independently examined by two pathologists. The 2001 Bethesda classification [29] was used for slide interpretation. Patients with cervical abnormalities (squamous intraepithelial lesions, SILS) were referred to the Colposcopy clinic for further follow-up.

\section{Papillocheck ${ }^{\mathrm{R}}$ HPV Genotyping}

500ul of PreserveCyt material was used for automated DNA extraction using BioRobot ${ }^{\circledR}$ M48 (Qiagen, Hilden, Germany) as described by the manufacturer. $5 \mathrm{ul}$ of purified DNA was used for HPV genotyping as per the PapilloCheck ${ }^{\mathrm{R}}$ test system (Greiner Bio-One, Germany) which simultaneously detects and identifies 24 different HPV genotypes; six low risk HPV types [6, 11, 40, 42, 43, $44,55]$ and 18 high risk potential high risk HPV types [16, $18,31,33,35,39,45,51,52,53,56,58,59,66,68,70,73$, 82]. To clarify, whilst all the latter HPV types are from the "high- risk clade" some of them are classified only as possible/probable carcinogens (types 53, 66, 68, 70, 73,82). This is due to lack of data on their transforming potential in model systems along with their low frequency [30]. In terms of the Greiner Papillocheck they are designated high risk and for consistency hereafter they will be referred to as high risk with the acceptance that this is not as well founded as for other HPV types.

\section{ICC Study Population}

Between 2008/9, 77 patients with histologically confirmed ICC, who had also undergone a HIV test, were recruited randomly at the KNH Department of Radiotherapy. This is the single largest center treating invasive ICC in Kenya and receives patients from all over the country. The median age was 42yrs (range 27-68yrs). Of the 77 patients recruited, 40 were HIV-negative while 37 were HIV-positive (of whom 15 patients were on HAART). After written informed consent, structured questionnaires were completed recording socio-demographics; examination under anesthesia, staging and cervical tumour biopsy was also done. CD4 counts were available for 34 out of the 37 HIVpositive patients and the range was $58-626 / \mathrm{mm}^{3}$ with a mean of $281 / \mathrm{mm}^{3}$. Formalin-fixed paraffin-embedded (FFPE) ICC specimens were shipped to the University of Manchester where DNA was extracted by BioRobot ${ }^{\circledR}$ M48 and endpoint PCR carried out for detection of HPV genotypes (see below).

\section{DNA Preparation from FFPE Material}

$6 \times 10$ micron serial FFPE ICC sections were cut per block using single-use disposable microtome blades. To further prevent cross contamination between blocks, disposable forceps were used for handling the sections and the middle 4 from each case were transferred directly into sterile microtubes. The first and last sections were stained using Haematoxylin and Eosin and reviewed to check that the material was representative of the diagnosis. In all cases this was found to be acceptable. DNA isolation was carried out using Qiagen Qiacube FFPE kits (Qiagen, Crawley, West Sussex, UK) according to the manufacturer's instructions.

\section{Endpoint PCR HPV Genotyping}

Hot-start, touch-down multiplex PCR was optimized with HPV specific primer sets targeting:- E6/7 and L1 regions of HPV16,18, 52,56 and 58; the E6/7 region of HPV53 and the L2 region HPV45 [3, 31-34] (Table 1). A multiplex PCR kit (Qiagen) was used as recommended by the manufacturer. All reactions were set up in duplicates, using a Veriti TM Thermal Cycler (Applied Biosystems, Paisley, UK) with the conditions and primers indicated in Table 1. The PCR products were separated by $2.5 \%$ agarose gel electrophoresis, stained with ethidium bromide and examined under UV light. Control GAPDH amplification was used as a positive control and was carried out using a $50 \mu 1$ reaction mixture containing $2 \mu 1$ of the DNA sample (50 ng), 2.5 units of BioTaq DNA polymerase (Bioline Ltd, London, UK), 0.2 $\mathrm{mM}$ NTPs, and $0.2 \mu \mathrm{M}$ of each primer in $10 \mathrm{mM}$ Tris-HCL 
Table 1. Primers and PCR Conditions Used in this Study

\begin{tabular}{|c|c|c|c|}
\hline Primer & Sequence & PCR Parameters & Amplicon Size (bp) \\
\hline HPV16E6/E7F & 5'-GTGGACCGGTCGATGTATGTCT-3' & \multirow{3}{*}{1} & 209 \\
\hline HPV16L1F & 5'-AGCACGGATGAATATGTTGCACG-3' & & \multirow{2}{*}{148} \\
\hline HPV16L1R & 5'-CCCTGTATTGTAATCCTGATACTTTAG-3' & & \\
\hline HPV18E6/E7R & 5'-GGCTTCACACTTACAACACA-3' & \multirow{3}{*}{2} & 286 \\
\hline HPV18L1/L2F & 5'-GATTATTTACAAATGTCTGCA-3' & & \multirow{2}{*}{118} \\
\hline HPV18L1/L2R & 5'-GCACAGTGTCACCCATAGTA-3' & & \\
\hline HPV45L2F & 5'-TGTTGGACATCACACCTACCGTGGA-3' & 3 & 205 \\
\hline GAPDH-F & 5'-CATTGACCTCAACTACATGGT-3' & \multirow{2}{*}{4} & \multirow{2}{*}{130} \\
\hline GAPDH-R & 5'-TCGCTCCTGGAAGATGGTGAT-3' & & \\
\hline
\end{tabular}

The PCR Parameters used, were as follows; (1) $95^{\circ} \mathrm{C}$ x $15 \min 8$ cycles: $94^{\circ} \mathrm{C} \mathrm{x} 1 \mathrm{~min}, 61^{\circ} \mathrm{C}-1{ }^{\circ} \mathrm{C} /$ cycle $\times 1.5 \mathrm{~min}, 72^{\circ} \mathrm{C} \times 1 \mathrm{~min} ; 37 \mathrm{cycles}: 94^{\circ} \mathrm{C} \mathrm{x} 1 \mathrm{~min}, 55^{\circ} \mathrm{C} \times 1 \mathrm{~min}, 72^{\circ} \mathrm{C} \times 1$ $\min ; 72^{\circ} \mathrm{C}$ x $7 \mathrm{~min},(2) 95^{\circ} \mathrm{C}$ x $15 \mathrm{~min} ; 8$ cycles: $94^{\circ} \mathrm{C} \mathrm{x} 1 \mathrm{~min}, 60^{\circ} \mathrm{C}-1^{\circ} \mathrm{C} /$ cycle x $1.5 \mathrm{~min}, 72 \mathrm{oC} \mathrm{x} 1 \mathrm{~min} ; 37 \mathrm{cycles}: 94^{\circ} \mathrm{C} \mathrm{x} 1 \mathrm{~min}, 53^{\circ} \mathrm{C} \mathrm{x} 1 \mathrm{~min}, 72^{\circ} \mathrm{C} \mathrm{x} 1 \mathrm{~min} ; 72^{\circ} \mathrm{C} \mathrm{x} 7 \mathrm{~min},(3)$ $95^{\circ} \mathrm{C}$ x $15 \mathrm{~min} ; 8$ cycles: $94^{\circ} \mathrm{C}$ x $1 \mathrm{~min}, 64^{\circ} \mathrm{C}-1^{\circ} \mathrm{C} /$ cycle x $1.5 \mathrm{~min}, 72^{\circ} \mathrm{C} \mathrm{x} 1 \mathrm{~min} ; 37$ cycles: $94^{\circ} \mathrm{C}$ x $1 \mathrm{~min}, 57^{\circ} \mathrm{C} \mathrm{x} 1 \mathrm{~min}, 72^{\circ} \mathrm{C} \mathrm{x} 1 \mathrm{~min} ; 72^{\circ} \mathrm{C} \mathrm{x} 7 \mathrm{~min}(4) 94^{\circ} \mathrm{C}$ x 5 min; 33 cycles: $94^{\circ} \mathrm{C} \times 25 \mathrm{~s}, 53^{\circ} \mathrm{C} \times 25 \mathrm{~s}, 72^{\circ} \mathrm{C} \times 25 \mathrm{~s} ; 72^{\circ} \mathrm{C} \times 7 \mathrm{~min}$

$\mathrm{pH} 8.3,50 \mathrm{mM} \mathrm{KCl}$, and $2.5 \mathrm{mM}$. All reactions were repeated four times.

\section{Statistical Analysis of Results}

In order to obtain a $95 \%$ confidence interval with a power of $80 \%$ and a ratio of HIV-positive to HIV-negative patients of $1: 1$, it was calculated that $102 \mathrm{HIV}$-positive and a similar number of HIV-negative patients were needed to detect significant differences between cervical dysplasia and HPV prevalence. We obtained 113 HIV-positive and 111 HIV-negative smear samples. The data was captured in an Access database and exported to SPSS version 16.0 for analysis after cleaning and validation. Proportions of participants with HPV infection was reported with 95\% confidence intervals by HIV WHO staging classifications. Statistical tests of significance were done using chi square with Yates correction or with Fisher's test if the expected frequencies in the cells were less than five. Further, crosstabulations were also used to access the distribution between HPV genotype and HIV status of the participants. This utilized Chi-Square non-parametric test to access statistical significance between the dependent (HPV) and risk Factors. A p-value of less than 0.05 was considered statistically significant.

\section{RESULTS}

\section{Cytological Findings in Cervical Smears}

The mean age of the LBC study subjects was 35.3 years with a range of 21 - 50 years. The majority of patients presented with cytologically normal pap smears $(79.0 \%)$, however, $4.5 \%$ were diagnosed as having HSIL, 11.2\% LSIL and $3.1 \%$ were reported with inflammatory changes as shown in Table 2 .

\section{HPV Genotyping in Cervical Smears Using the Papillocheck $^{\mathrm{R}}$ System}

Of the 224 LBC specimens analyzed for HPV types one had an invalid result (i.e did not amplify with the Papillocheck "house-keeping gene" Adenosine deaminase1, ADAT1 ), while 121 out of the remaining $223(55 \%)$ had at least one HPV type with seven being the highest number detected in any one individual. All twenty-four genotypes covered by Papillocheck were encompassed by the sample set. High risk HPV infection was more prevalent in HIV-positive individuals with $68 / 113$ $(60.2 \%)$ of HIV positive women co-infected with high risk HPV, as compared to only $37 / 110$ (33.6\%) among HIV negative women (Fig. 2). The commonest high-risk HPV detected was type 56 with a total of twenty-three infections whereas type 18 was the least common seen in only three infections (Table 4). The other vaccine targeted HPV (type 16) accounted for only $8.4 \%$ of the women found to be infected with a high-risk HPV. Indeed types 56, 52 and 58 were far more common. HPV genotypes 52, 58, 53 and 68 in addition to lowrisk HPV 44/55 showed a significant association with HIV (p values ranging from 0.04 to 0.002 ; see Table 4). Analysis of the linkage between HIV, HPV and abnormal cervical cytology showed significant associations as indicated in Table $\mathbf{3}$. Similarly, most HPV infections occurred in more advanced HIV stages as seen in Fig. (1A). Analysis of HPV types and immune status as measured by CD4 counts was not evaluated due to the small numbers of samples involved. HIV infection was decidedly associated with multiple HPV infections and infection with high risk genotypes $(\mathrm{p}<0.006, \mathrm{p}<0.01)$ (Table 4, Fig. 1A, B). When focussing solely on those women having smears with abnormal cytology there was a significant increase in the detection of all high-risk types and this was more pronounced in HIV-positive women. However the number of patients involved (ranged between 3-23 positive samples per HPV type) was not 
Table 2. HIV Infection and Grade of Cervical Cytology in LBC Samples

\begin{tabular}{|c|c|c|c|}
\hline Cytology & HIV+ve (n=113) & HIV-ve (n=111) & Total (n=224) \\
\hline \hline Normal & $76 / 67.3 \%$ & $101 / 91.0 \%$ & $177 / 79.0 \%$ \\
\hline LSIL/CIN I & $19 / 16.8 \%$ & $6 / 5.4 \%$ & $25 / 11.2 \%$ \\
\hline HSIL/CIN II/III & $9 / 8.0 \%$ & $1 / 0.9 \%$ & $10 / 4.5 \%$ \\
\hline ASCUS/AGUS & $4 / 3 \mathrm{hg} 5 \%$ & $1 / 0.9 \%$ & $5 / 2.2 \%$ \\
\hline Inflammatory & $5 / 4.4 \%$ & $2 / 1.8 \%$ & $7 / 3.1 \%$ \\
\hline Total & $113 / 50.4 \%$ & $111 / 49.6 \%$ & $224 / 100 \%$ \\
\hline
\end{tabular}

HIV+ve women had 4 times higher prevalence of abnormal cervical cytology as compared to HIV-ve ( $28.3 \%$ Vs $7.2 \%$ respectively) with p value $<0.001$

sufficient to draw conclusions regarding any differences in the prevalence of discrete HPV types between HIV-positive and -ve smears with abnormal cytology.
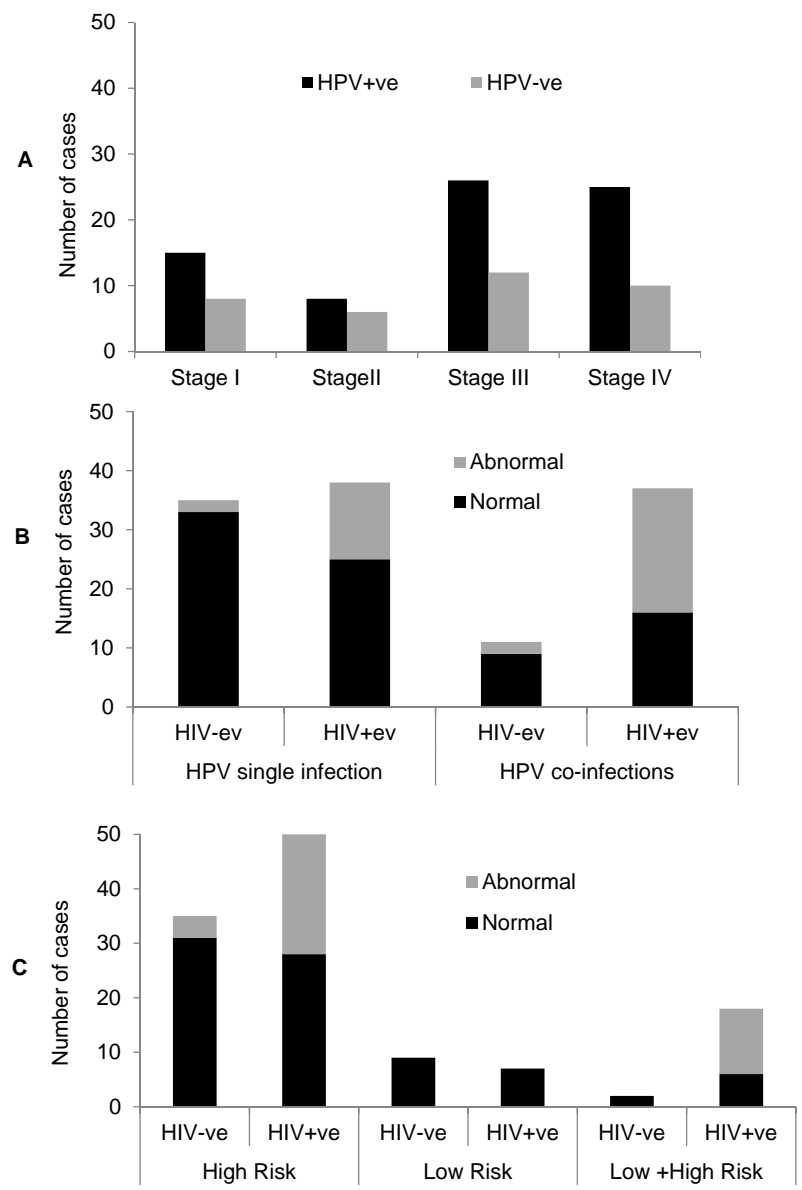

Fig. (1). HIV is associated with increased numbers of both single and multiple HPV infections and abnormal cytology in LBC Samples. (A) Illustrates the association between HPV infection and HIV WHO staging. It can be seen that most HPV infections occurred in advanced stages of III \& IV although this was not judged to be significantly different $(p=0.09)$. (B) Shows the association between HIV \& HPV co-infections and abnormal cytology $(\mathrm{n}=121)$. HIV infection was highly associated with multiple HPV infections ( $p<0.006$ ), and was a significant factor for abnormal cervical cytology in both single and multiple HPV infections. (C) Shows the association between HIV and HPV highrisk versus low-risk genotypes $(n=121)$. Infection with high-risk HPV types and HIV infection was highly associated with abnormal cervical cytology $(\mathrm{p}<0.01)$.

\section{HPV Genotyping in ICC's by Endpoint Multiplex PCR}

Since the Papillocheck ${ }^{\mathrm{R}}$ method is more suitable for analysing LBC derived DNA [35], rigorous endpoint multiplex PCR was used to analyse DNA extracted from ICC tissues for the presence of HPV types 16, 18, 53, 52, 56, 58 and 45 . These particular types were selected firstly on the basis of having previously been highly associated with ICC $[16,18$ and 45) and secondly those that we most commonly found in LBC samples (see Fig. 2). Two different sets of primers were used for the analysis of HPVs 16, 18, 52, 56 and 58, whereas a single set of primers was used for HPV 53 and 45. A total of 12 samples were positive for HPV16 in HIV-positive ICC's and there was good agreement between primer sets targeting the E6/7 and L1 regions with only one sample positive for E6/7 and not L1. A total of 22 samples (55\%) were positive for HPV16 in HIV-negative ICC's of which 16 were positive with both primer sets, 5 for E6/7 and 1 for L1. A total of 7 (19\%) samples were positive for HPV18 in HIV-positive ICC's of which 3 were positive for both primer sets and 4 for L1. A total of 14 (35\%) samples were positive for HPV18 in HIV-negative ICC's of which 7 were positive for both primers sets and 7 with L1. HPV45 was detected in $6 \mathrm{HIV}$-positive and $1 \mathrm{HIV}$-negative ICC whereas HPV53 was not found in any (Fig. 3 and Table 5). These results indicate that HPV45 infection was significantly associated with HIV positivity in ICC samples $(p<0.05)$ whereas in HIV-negative women we observed an increase in HPV16 and HPV16/18 infections ( $<<0.01, \mathrm{p}<0.05)$.

\section{Socio-Demographic Factors}

There was a statistically significant association $(p<0.011)$ between HPV infection and women who had multiple sexual partners (Defined as $>5$ over lifetime). However, even though HPV infection was more common in single and separated/divorced women as compared to the married/widowed cohort, this association did not achieve statistical significance, $\mathrm{p}=0.43$ (data not shown). The following socio-demographics did not show significant statistical association with HPV infection: religion, educational level, patient occupation, socio-economic status, parity, age of menarche or sexual debut, or residing in urban versus rural Kenya.

\section{DISCUSSION}

The initial objective of this study was to profile the HPV types found in cervical smears from HIV-positive and HIVnegative Kenyan women. However, when this was done it was noticed that the incidence of HPV16 and 18 was much lower than expected which prompted analysis of the 77 


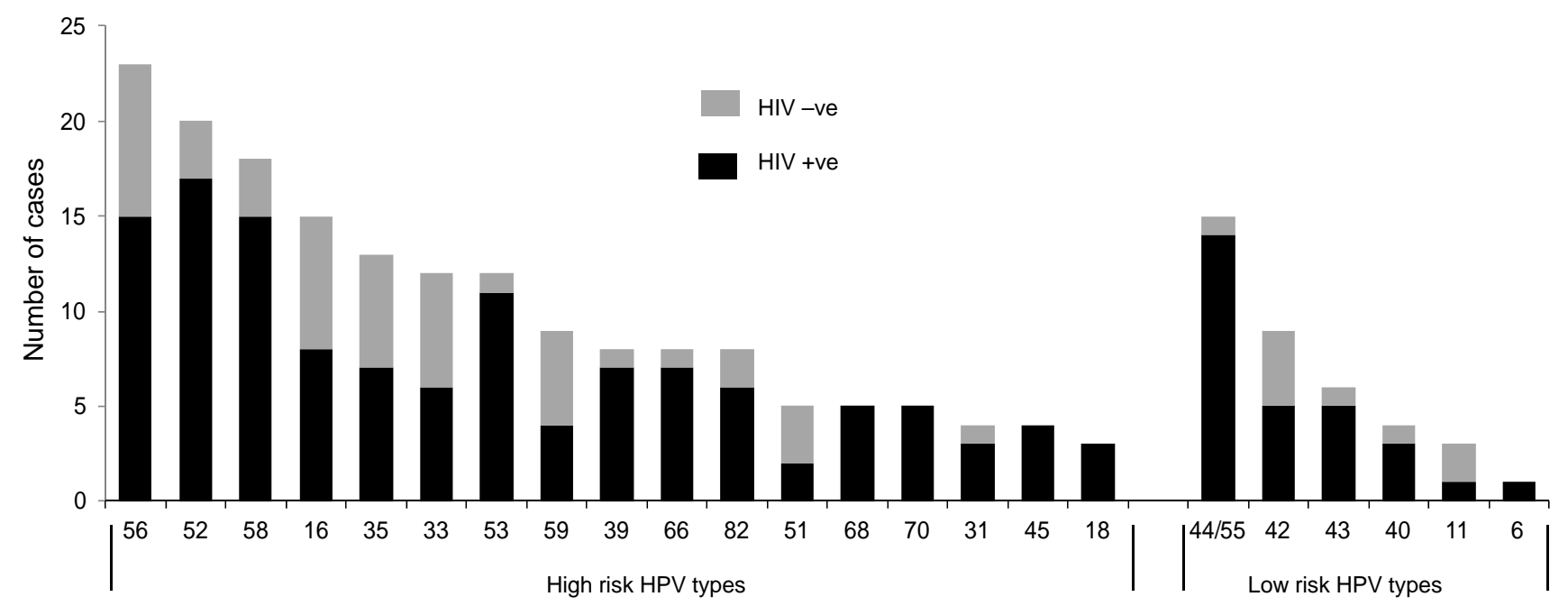

Fig. (2). HIV infection is associated with specific HPV types. Comparative numbers of each HPV type detected in HIV-positive and HIVnegative women are shown with high-risk on the left and low-risk on the right. Almost all HPV types tested were more prevalent in HIVpositive patients compared to HIV-negative patients.

Table 3. Associations Between HIV, HPV and Abnormal Cervical Cytology in LBC Samples

\begin{tabular}{|c|c|c|c|c|c|}
\hline \multirow{2}{*}{ HIV Status } & \multicolumn{2}{|c|}{ HPV } & \multicolumn{2}{|c|}{ Cytology } & \multirow{2}{*}{ Total } \\
\hline & $+v e$ & -ve & Normal & Abnormal & \\
\hline HIV +ve & $75 / 66.4 \%$ & $38 / 33.6 \%$ & $61 / 54.0 \%$ & $52 / 46.0 \%$ & $113 / 50.4 \%$ \\
\hline HIV -ve & $46 / 41.4 \%$ & $* 64 / 58.6 \%$ & $97 / 87.4 \%$ & $14 / 12.6 \%$ & $111 / 49.6 \%$ \\
\hline Total & $121 / 54.0 \%$ & $102 / 45.5 \%$ & $158 / 70.5 \%$ & $66 / 29.5 \%$ & $224 / 100 \%$ \\
\hline P-value & \multicolumn{2}{|c|}{0.001} & \multicolumn{2}{|c|}{0.0001} & \\
\hline
\end{tabular}

positive patient was more likely to be HPV positive and also more likely to have abnormal cervical cytology as compared to a HIV negative patient ( $\mathrm{n}=224)$.

FFPE ICC samples we had available from the same geographical location. These results showed that whilst only a low prevalence of HPV16 and 18 was found in LBC's from HIV-positive and negative women, this was markedly increased in ICCs, particularly from those women not infected with HIV. In HIV-positive ICC samples on the other hand type 45 , which is a type 18 related HPV, showed the highest prevalence. We also identified an unusual association between specific types of HPV and HIV in cervical smears (i.e. types 52, 53, 58, 68 with p-values $<0.05$; Table 4) and the finding that many of the atypical HPV types that predominate in LBC samples (i.e. 52, 53, 56 and 58) were completely absent in the ICC specimens (data not shown).

How do our results compare to previously published work in this area? When focussing on ICC alone a recent multicentre study by De Vuyst et al. [36] in patients recruited from Kenya and South Africa, reported a modest excess of HPV18 in HIV-positive compared to HIV-negative cases (Prevalence ratio $(\mathrm{PR})=1.9,95 \%$ confidence interval (CI): 1.0-3.7) [28]. However, the combined HPV16 and/or 18 prevalence, was similar in HIV-positive $(66.7 \%)$ and HIV-negative cases of ICC (69.1\%) (PR $=1.0$, 95\% CI: $0.9-$ 1.2 ) and no significant difference was found for other HPV types. In an earlier study also carried out on Kenyan women with ICC and varied HIV status, HPV16 was shown to be the most common type detected in both groups $(41.2 \%$ in HIVpositive and $43.8 \%$ in HIV-negative) with HPV16 and/or 18 being found in $64.7 \%$ versus $60.1 \%$, respectively [37]. The most frequently found HPV types after this were HPV 52, 35, 45 and 56 among HIV-positive women, and HPV45, 35 and 52 among HIV-negative women. Therefore, whilst we are in agreement with both of these studies in that our data supports the importance of HPV16/18 in cervical carcinogenesis in Kenyan women, in contrast we have found a reduced incidence of types 16 and 18 in ICC's from HIV-positive (45\%) when compared to HIV-negative women $(80 \%)$ and an association of HPV45 with HIV-positive ICC.

The HPV typing results on LBC samples (Fig. 1B, C) demonstrate that HIV infection is highly associated with multiple HPV infections and with specific high-risk HPV types $(\mathrm{p}<0.006$ and 0.01 respectively). Analogous observations have also been made from previous studies in Kenya [38] which are conistent with other reported HPV screens carried out in Africa [39] and elsewhere in the world where detection of unusual patterns of non-16/18 HPV types in HIV infected individuals is a common feature [38, 40-43]. This difference has been attributed to the fact that clearance of HPV16 is less dependent on an individual's immune status than clearance of other high-risk types [44].

Whilst collectively the results seem to suggest that different types of HPV may behave differently in HIVpositive individuals and that this in turn may influence their role in the aetiology of both premalignant disease, and 
Table 4. Association Between Different HPV Genotypes and HIV in LBC Samples

\begin{tabular}{|c|c|c|c|c|}
\hline \multirow{2}{*}{ Genotype } & Total & HIV +ve & HIV-ve & \multirow{2}{*}{ P-Value } \\
\hline & Frequency/\% & Frequency/\% & Frequency/\% & \\
\hline \multicolumn{5}{|l|}{ High Risk } \\
\hline 56 & $23 / 10.3 \%$ & $15 / 65.2 \%$ & $8 / 34.8 \%$ & 0.203 \\
\hline 52 & $20 / 8.9 \%$ & $17 / 85.0 \%$ & $3 / 15.0 \%$ & 0.003 \\
\hline 58 & $18 / 8.0 \%$ & $15 / 83.3 \%$ & $3 / 16.7 \%$ & 0.009 \\
\hline 16 & $15 / 6.7 \%$ & $8 / 53.3 \%$ & $7 / 46.7 \%$ & 0.586 \\
\hline 35 & $13 / 5.8 \%$ & $7 / 53.8 \%$ & $6 / 46.2 \%$ & 0.583 \\
\hline 53 & $12 / 9.9 \%$ & $11 / 91.7 \%$ & $1 / 8.3 \%$ & 0.009 \\
\hline 33 & $12 / 5.4 \%$ & $6 / 50 \%$ & $6 / 50 \%$ & 0.599 \\
\hline 59 & $10 / 8.3 \%$ & $5 / 50 \%$ & $5 / 50 \%$ & 0.521 \\
\hline 66 & $9 / 7.4 \%$ & $7 / 77.8 \%$ & $2 / 22.2 \%$ & 0.058 \\
\hline 39 & $9 / 7.4 \%$ & $7 / 77.8 \%$ & $2 / 22.2 \%$ & 0.058 \\
\hline 82 & $9 / 7.4 \%$ & $6 / 66.7 \%$ & $3 / 33.3 \%$ & 0.243 \\
\hline 68 & $6 / 5.0 \%$ & $6 / 100 \%$ & $0 / 0.0 \%$ & 0.040 \\
\hline 51 & $5 / 4.1 \%$ & $2 / 40 \%$ & 3.6 & 0.499 \\
\hline 70 & $5 / 4.1 \%$ & $5 / 100 \%$ & $0 / 0.0 \%$ & 0.068 \\
\hline 45 & $4 / 1.8 \%$ & $4 / 100 \%$ & $0 / 0.0 \%$ & 0.083 \\
\hline 31 & $4 / 3.3 \%$ & $3 / 75 \%$ & $1 / 25 \%$ & 0.414 \\
\hline 18 & $3 / 1.3 \%$ & $3 / 100 \%$ & $0 / 0.0 \%$ & 0.137 \\
\hline \multicolumn{5}{|l|}{ Low Risk } \\
\hline $44 / 55$ & $15 / 13.2 \%$ & $14 / 93.3 \%$ & $1 / 6.7 \%$ & 0.002 \\
\hline 42 & $8 / 6.6 \%$ & $5 / 62.5 \%$ & $3 / 37.55$ & 0.966 \\
\hline 43 & $6 / 5.0 \%$ & $5 / 83.3 \%$ & $1 / 16.7 \%$ & 0.115 \\
\hline 11 & $5 / 4.1 \%$ & $2 \% / 50 \%$ & $2 / 50 \%$ & 0.406 \\
\hline 40 & $4 / 3.3 \%$ & $3 / 75 \%$ & $1 / 255$ & 0.414 \\
\hline 6 & $1 / 0.8 \%$ & $1 / 100 \%$ & $0 / 0.0 \%$ & 0.616 \\
\hline
\end{tabular}

Although HPV infection was generally more prevalent in HIV +ve than in HIV -ve patients, genotypes 52, 58, 53, 68 and 44/55 showed the most significant association with HIV (Significant $\mathrm{p}$ values shown in bold). Total sample number $\mathrm{n}=224$.

potentially ICC, there are data which refute this. For example a recently published study on Mozambique women concluded that within the limitations of their study the relative importance of HPV types in cervical cancer did not appear to be greatly modified by HIV infection [45].

Our comparison of the types of high-risk HPV found in smears to those detected in ICC's showed that, although some of the commonest high-risk HPVs found in HIVpositive LBC smears were also found in HIV-positive ICC's, others were completely absent (e.g. type 53, 52, 56 \& 58). This indicates that even though these atypical HPV types predominate in LBCs with normal and abnormal cytology, they lose this dominance to HPV16 and/or HPV18, in addition to type 45 , in the progression towards ICC. What is the explanation for this and how does it relate to cervical carcinogenesis in HIV-positive women? One potential possibility is type competition where if two individual types are competitors they cannot co-exist. For instance type 33 has been postulated to have a competitive advantage over other HPV types [46]. In addition infections with certain HPV types are dependent upon the existence of others so clearance of one may have an effect on the natural history of another [47].

Taking these possibilities into account we hypothesise that HIV facilitates infection with specific types of HPV some of which may promote (HPV-p types) or inhibit (HPV$i$ types) progression to cervical carcinogenesis in any single individual. Indeed, mechanistically HPV-i types could persist more readily as episomes thereby producing functional E2 proteins which in turn negatively regulate the transcription of oncogenic E6/E7 from integrated HPV-p types. The latter as a consequence of integration would have a disrupted, thus non functional, E2 open reading frame [48]. Clearly this hypothesis could be readily tested by analysing the integration status of the various HPV types present in cervical smears from a cohort of HIV-positive and HIV- 

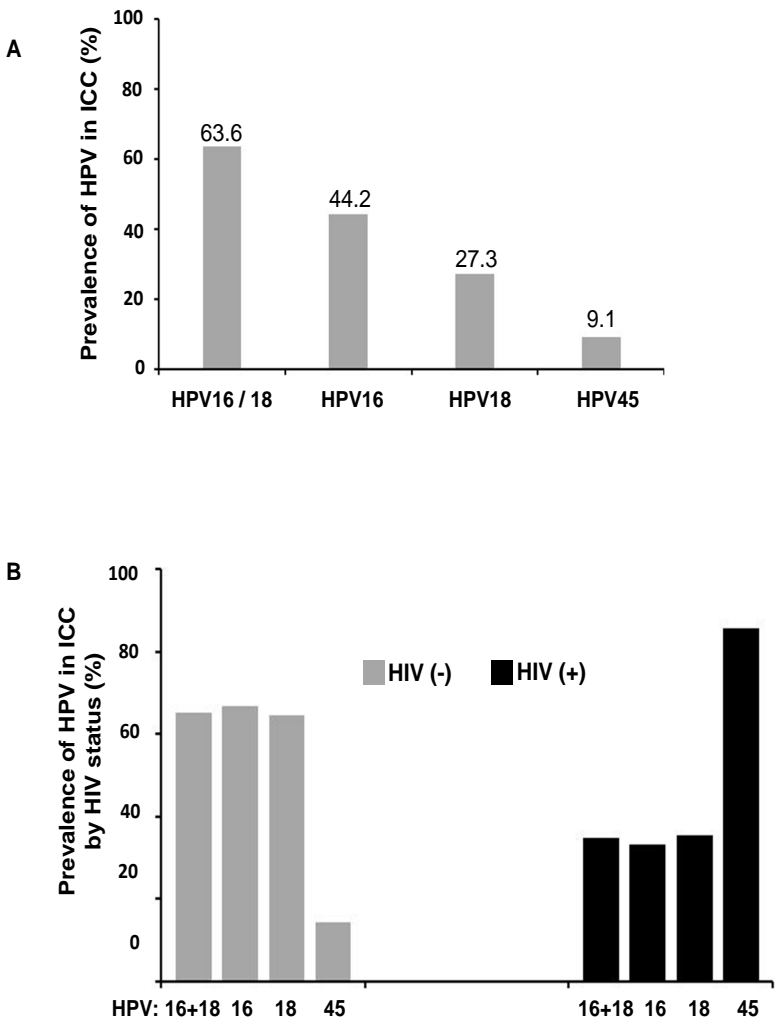

Fig. (3). Statistical analysis of HPV types detected in HIVpositive and HIV-negative ICC's: There was significant association between HIV infection and HPV types 16 and $45(\mathrm{p}<$ 0.046-0.0019). HIV-negative women had a higher prevalence of HPV16 and HPV16/18 infections when compared to HIV-positive women $(\mathrm{p}<0.01, \mathrm{p}<0.05)$.

negative Kenyan women and then assessing the overall expression levels of E2 and E6/E7. This is supported by a previous study on ICC which showed that individual highrisk HPV types had marked differences in their frequency of integration into host DNA [49] and in vitro tests which suggested high-risk HPV types had an increased ability to integrate into host DNA when compared with low-risk types [50]. Indeed, in light of our observations regarding types 52 and 58, it is significant that both of these types are known to integrate infrequently [51]. However, a recent study on the functional interactions of E2 proteins from different HPV types has shown that these are highly complex and differ from each other [52]. Consequently, although it is certainly possible that E2 proteins, from some HPV types, may effect the transforming potential of other high-risk types present, the mechanism for this effect is likely to be complex.

The concept of inhibitory and unrestrictive HPV types might also offer another potential explanation for the paradox of why HIV increases multiple HPV infections and associated cervical dysplasia yet it does not produce a large increase in ICC. Some authors suggest that progression rates towards cervical cancer are the same irrespective of HIV status and that it is persistence of HPV which is altered [15]. In contrast accelerated progression of pre-malignant cervical lesions to ICC has been reported in Kenyan HIV infected women [12]. Indeed, the observation that HIV infected women present with ICC 10-15 years earlier than HIV negative women has also been reported in two previous studies both carried out in S. Africa [53, 54]. Interestingly, our finding of an association of HPV45 with HIV may fit with these latter studies since the mean age of patients with cervical cancer is the lowest for HPV45 (46.8 years), followed by HPV18 (48.2 years) and HPV16 (50.0 years) suggesting quicker progression to ICC in the presence of certain HPVs which in turn may be related to their frequency of integration e.g. $83 \%$ for type 45 and $55-80 \%$ for type 16 [49]. However, HPV typing was not performed in the either of the S. African or the 2003 Kenyan study so this remains speculative.

Our observation that HIV infection was significantly associated with abnormal cervical cytology, (Table 2) is consistent with studies carried out in Kenya [55] and in other African and non-African locations [5, 56-60]. Furthermore, our data also supports the previously well documented association between HIV and HPV in LBC samples (Table 3) $[58,59,61,62]$.

In the current study, most HPV infections among HIVpositive women were found in HIV WHO late stages III and IV AIDS implying that HPV infections were associated with a reduced level of immunity (Fig. 1A). The limits to our study were the modest sample size and lack of follow-up which restricts a full evaluation of the significance of the unusual HPV genotypes found in smears from the HIVpositive population. Another limitation is the lack of data concerning age of acquisition of HIV infection since it is possible this may have occurred too late in life for some of the women in our study to influence the natural history of the HPV types involved in cervical carcinogenesis. Indeed the timing of HIV infection provides a potential explanation for why, the results of the De Vuyst et al. [37] may differ from ours since they did not show any significant difference in HPV types in HIV-positive versus negative ICCs from Kenyan women. In the De Vuyst patient cohort over half of the HIV infected women had a CD4 count of above 500/L leading them to speculate a relatively recent HIV infection. HAART treatment will of course influence the CD4 counts but as no statistics are available for their patient cohort it is likely that very few women, if any, were undergoing such treatment at the time of this study. In order to resolve the issue there is undoubtedly a need to conduct a larger study

Table 5. Association Between HPV Genotypes and HIV Status in ICC Samples

\begin{tabular}{|c|c|c|c|c|c|c|c|c|c|c|}
\hline & HPV16 (-) & HPV16 (+) & HPV18 (-) & HPV18 (+) & HPV16/18 (-) & HPV16/18 (+) & HPV45 (-) & HPV45 (+) & HPV53 (-) & HPV53 (+) \\
\hline HIV (-) & 18 & 22 & 26 & 14 & 8 & 32 & 34 & 6 & 40 & 0 \\
\hline p value & \multicolumn{2}{|c|}{0.046} & \multicolumn{2}{|c|}{0.113} & \multicolumn{2}{|c|}{0.0019} & \multicolumn{2}{|c|}{0.036} & \multicolumn{2}{|c|}{ - } \\
\hline
\end{tabular}


on ICC's from Kenyan women infected with HIV at an early age (i.e. ideally women who have acquired an oncogenic HPV type after HIV infection and have survived long enough to develop ICC).

The major strength of our study is the fact that we have been able to compare, albeit in a pilot way, the HPV types of both general LBC samples and ICC material from the same population of women in a single geographical location. In addition, knowledge of HIV status has allowed us to look for any potential linkage between the two viruses.

Considered collectively with previous work our findings indicate that the ability of current vaccines to protect against HPV 16 and 18 related ICC may be similar in HIV-positive and HIV-negative women in Kenya, provided vaccination is administered before sexual debut and that protection is sustained after HIV infection [27, 28, 37]. Both current vaccines Gardasil and Cervarix afford cross protection against type $45 \mathrm{HPV}$ with Cervarix suggested to offer the higher degree of effectiveness [63]. That the vaccines have this property is important in light of our findings with the HPV 45 and HIV-positive ICC women.

What is obvious from our own and other published studies are that the relationship between HPV types, HIV positivity and progression to ICC is not straightforward and the picture will only become clearer with greater acquisition of data. This issue clearly needs to be resolved since it has direct bearing on the potential benefits which may result from implementing HPV vaccination in HIV-positive women in African countries. Furthermore, it could also provide improved guidance on the likelihood of progression to cancer and may even indicate novel approaches for the prevention of this disease. More studies are urgently needed to clarify the rationale behind the varied observations and hypotheses and to strengthen ICC as a public health priority in Kenya.

\section{CONFLICT OF INTEREST}

The authors confirm that this article content has no conflict of interest.

\section{ACKNOWLEDGEMENTS}

The work described was funded by charitable donations from the Humane Research Trust, The Janice Cholerton Post Graduate Support Fund, The Caring Cancer Trust, The Cancer Prevention Research Trust and United in Cancer UK. Post graduate student IOM was part funded by the International Atomic Energy Agency and Wellbeing of Women. The funders had no role in study design, data collection and analysis, decision to publish, or preparation of the manuscript.

\section{REFERENCES}

[1] Bosch FX, Lorincz A, Munoz N, Meijer CJ, Shah KV. The causal relation between human papillomavirus and cervical cancer. $\mathrm{J}$ Clin Pathol 2002; 55(4): 244-65.

[2] zur Hausen H. Papillomavirus infections--a major cause of human cancers. Biochim Biophys Acta 1996; 1288(2): F55-78.

[3] Walboomers JM, Jacobs MV, Manos MM, et al. Human papillomavirus is a necessary cause of invasive cervical cancer worldwide. J Pathol 1999; 189(1): 12-9.

[4] Parkin DM, Pisani P, Ferlay J. Estimates of the worldwide incidence of 25 major cancers in 1990. Int J Cancer 1999; 80(6): 827-41.
[5] Temmerman M, Tyndall MW, Kidula N, Claeys P, Muchiri L, Quint W. Risk factors for human papillomavirus and cervical precancerous lesions, and the role of concurrent HIV-1 infection. Int J Gynaecol Obstet 1999; 65(2): 171-81.

[6] CDC. 1993 revised classification system for HIV infection and expanded surveillance case definition for AIDS among adolescents and adults. MMWR Recomm Rep 1992; 41(RR-17): 1-19.

[7] WHO. WHO case definitions of HIV for surveillance and revised clinical staging and immunological classification of HIV-related disease in adults and children2007: Available from: http://www.who.int/hiv/pub/guidelines/HIVstaging150307.pdf.

[8] Smith-McCune KK, Shiboski S, Chirenje MZ, et al. Type-specific cervico-vaginal human papillomavirus infection increases risk of HIV acquisition independent of other sexually transmitted infections. PLoS One 2010; 5(4): e10094.

[9] Schiffman M, Herrero R, Desalle R, et al. The carcinogenicity of human papillomavirus types reflects viral evolution. Virology 2005; 337(1): 76-84.

[10] Strickler HD, Burk RD, Fazzari M, et al. Natural history and possible reactivation of human papillomavirus in human immunodeficiency virus-positive women. J Natl Cancer Inst 2005; 97(8): 577-86.

[11] La Ruche G, Ramon R, Mensah-Ado I, et al. Squamous intraepithelial lesions of the cervix, invasive cervical carcinoma, and immunosuppression induced by human immunodeficiency virus in Africa. Dyscer-CI Group. Cancer 1998; 82(12): 2401-8.

[12] Gichangi PB, Bwayo J, Estambale B, et al. Impact of HIV infection on invasive cervical cancer in Kenyan women. AIDS 2003; 17(13): 19638.

[13] Hawes SE, Critchlow CW, Faye Niang MA, et al. Increased risk of high-grade cervical squamous intraepithelial lesions and invasive cervical cancer among African women with human immunodeficiency virus type 1 and 2 infections. J Infect Dis 2003; 188(4): 555-63.

[14] Rabkin CS, Biggar RJ, Baptiste MS, Abe T, Kohler BA, Nasca PC. Cancer incidence trends in women at high risk of human immunodeficiency virus (HIV) infection. Int J Cancer 1993; 55(2): 20812.

[15] Sun XW, Kuhn L, Ellerbrock TV, Chiasson MA, Bush TJ, Wright TC, Jr. Human papillomavirus infection in women infected with the human immunodeficiency virus. N Engl J Med 1997; 337(19): 1343-9.

[16] Franceschi S, Dal Maso L, Pezzotti P, et al. Incidence of AIDS-defining cancers after AIDS diagnosis among people with AIDS in Italy, 19861998. J Acquir Immune Defic Syndr 2003; 34(1): 84-90.

[17] Franceschi S, Dal Maso L, Arniani S, et al. Linkage of AIDS and cancer registries in Italy. Int J Cancer 1998; 75(6): 831-4.

[18] Dal Maso L, Serraino D, Franceschi S. Epidemiology of AIDS-related tumours in developed and developing countries. Eur J Cancer 2001; 37(10): 1188-201.

[19] Adjorlolo-Johnson G, Unger ER, Boni-Ouattara E, et al. Assessing the relationship between HIV infection and cervical cancer in Cote d'Ivoire: a case-control study. BMC Infect Dis 2010; 10: 242.

[20] Schmauz R, Okong P, de Villiers EM, et al. Multiple infections in cases of cervical cancer from a high-incidence area in tropical Africa. Int $\mathrm{J}$ Cancer 1989; 43(5): 805-9.

[21] ter Meulen J, Eberhardt HC, Luande J, et al. Human papillomavirus (HPV) infection, HIV infection and cervical cancer in Tanzania, east Africa. Int J Cancer 1992; 51(4): 515-21.

[22] Stein L, Urban MI, O'Connell D, et al. The spectrum of human immunodeficiency virus-associated cancers in a South African black population: results from a case-control study, 1995-2004. Int J Cancer 2008; 122(10): 2260-5.

[23] Kahesa C, Mwaiselage J, Wabinga HR, Ngoma T, Kalyango JN, Karamagi CA. Association between invasive cancer of the cervix and HIV-1 infection in Tanzania: the need for dual screening. BMC Public Health 2008; 8: 262.

[24] Odida M, Sandin S, Mirembe F, Kleter B, Quint W, Weiderpass E. HPV types, HIV and invasive cervical carcinoma risk in Kampala, Uganda: a case-control study. Infect Agent Cancer 2011; 6(1): 8.

[25] Ali-Risasi C, Praet M, Van Renterghem L, et al. Human papillomavirus genotype profile in Kinshasa, Democratic Republic of the Congo: implications for vaccination. Med Trop (Mars) 2008; 68(6): 617-20.

[26] McKenzie ND, Kobetz EN, Hnatyszyn J, Twiggs LB, Lucci JA, 3rd. Women with HIV are more commonly infected with non-16 and -18 high-risk HPV types. Gynecol Oncol 2010; 116(3): 572-7.

[27] Louie KS, de Sanjose S, Mayaud P. Epidemiology and prevention of human papillomavirus and cervical cancer in sub-Saharan Africa: a comprehensive review. Trop Med Int Health 2009; 14(10): 1287-302. 
[28] Vuyst HD, Ndirangu G, Moodley M, et al. Prevalence of human papillomavirus in women with invasive cervical carcinoma by HIV status in Kenya and South Africa. Int J Cancer 2011; 131(4): 949-55.

[29] Smith AE, Sherman ME, Scott DR, et al. Review of the Bethesda System atlas does not improve reproducibility or accuracy in the classification of atypical squamous cells of undetermined significance smears. Cancer 2000; 90(4): 201-6.

[30] Bouvard V, Baan R, Straif K, et al. A review of human carcinogens-Part B: biological agents. Lancet Oncol 2009; 10(4): 321-2.

[31] Tadokoro K, Akutsu Y, Tanaka K, et al. Comparative quantitative analysis of 14 types of human papillomavirus by real-time polymerase chain reaction monitoring Invader reaction (Q-Invader assay). Diagn Microbiol Infect Dis 2010; 66(1): 58-64.

[32] Dictor M, Warenholt J. Single-tube multiplex PCR using type-specific E6/E7 primers and capillary electrophoresis genotypes 21 human papillomaviruses in neoplasia. Infect Agent Cancer 2011;6(1): 1 .

[33] Mori S, Nakao S, Kukimoto I, Kusumoto-Matsuo R, Kondo K, Kanda $\mathrm{T}$. Biased amplification of human papillomavirus DNA in specimens containing multiple human papillomavirus types by PCR with consensus primers. Cancer Sci 2011; 102(6): 1223-7.

[34] Nishiwaki M, Yamamoto T, Tone S, et al. Genotyping of human papillomaviruses by a novel one-step typing method with multiplex PCR and clinical applications. J Clin Microbiol 2008; 46(4): 1161-8.

[35] Dehn D, Torkko KC, Shroyer KR. Human papillomavirus testing and molecular markers of cervical dysplasia and carcinoma. Cancer 2007; 111(1): 1-14.

[36] Vuyst HD, Ndirangu G, Moodley M, et al. Prevalence of human papillomavirus in women with invasive cervical carcinoma by HIV status in Kenya and South Africa. Int J Cancer 2012; 131(4): 949-55.

[37] De Vuyst H GP, Estambale B, Njuguna E, Franceschi S, Temmerman M. Human papillomavirus types in women with invasive cervical carcinoma by HIV status in Kenya. Int J Cancer 2008; 123(5): 1224-5.

[38] Blossom DB, Beigi RH, Farrell JJ, et al. Human papillomavirus genotypes associated with cervical cytologic abnormalities and HIV infection in Ugandan women. J Med Virol 2007; 79(6): 758-65.

[39] Sahasrabuddhe VV, Mwanahamuntu MH, Vermund $\mathrm{SH}$, et al. Prevalence and distribution of HPV genotypes among HIV-infected women in Zambia. Br J Cancer 2007; 96(9): 1480-3.

[40] Firnhaber C, Van Le H, Pettifor A, et al. Association between cervical dysplasia and human papillomavirus in HIV seropositive women from Johannesburg South Africa. Cancer Causes Control 2010; 21(3): 43343

[41] Joshi SN, Gopalkrishna V, Kumar BK, et al. Cervical squamous intraepithelial changes and human papillomavirus infection in women infected with human immunodeficiency virus in Pune, India. J Med Virol 2005; 76(4): 470-5.

[42] Singh DK, Anastos K, Hoover DR, et al. Human papillomavirus infection and cervical cytology in HIV-infected and HIV-uninfected Rwandan women. J Infect Dis 2009; 199(12): 1851-61.

[43] Clifford GM GM, Franceschi S. HPV and HIV Study Group. Human papillomavirus types among women infected with HIV: a metaanalysis. AIDS 2006; 20: 2337-44.

[44] Strickler HD PJ, Shah KV, Anastos K, et al. Human papillomavirus type 16 and immune status in human immunodeficiency virusseropositive women. J Natl Cancer Inst 2003; 95: 1062-71.

[45] Naucler P, Mabota da Costa F, da Costa JL, Ljungberg O, Bugalho A, Dillner J. Human papillomavirus type-specific risk of cervical cancer in a population with high human immunodeficiency virus prevalence: case-control study. J Gen Virol 2011; 92(Pt 12): 2784-91.
[46] Mejlhede N, Pedersen BV, Frisch M, Fomsgaard A. Multiple human papilloma virus types in cervical infections: competition or synergy? APMIS 2010; 118(5): 346-52.

[47] Merikukka M, Kaasila M, Namujju PB, et al. Differences in incidence and co-occurrence of vaccine and nonvaccine human papillomavirus types in Finnish population before human papillomavirus mass vaccination suggest competitive advantage for HPV33. Int $\mathbf{J}$ Cancer 2011; 128(5): 1114-9.

[48] Romanczuk H, Howley PM. Disruption of either the E1 or the E2 regulatory gene of human papillomavirus type 16 increases viral immortalization capacity. Proc Natl Acad Sci USA 1992; 89(7): 315963.

[49] Vinokurova S, Wentzensen N, Kraus I, et al. Type-dependent integration frequency of human papillomavirus genomes in cervical lesions. Cancer Res 2008; 68(1): 307-13.

[50] Kessis TD, Connolly DC, Hedrick L, Cho KR. Expression of HPV16 E6 or E7 increases integration of foreign DNA. Oncogene 1996; 13(2): 427-31.

[51] Ho CM, Chien TY, Huang SH, Lee BH, Chang SF. Integrated human papillomavirus types 52 and 58 are infrequently found in cervical cancer, and high viral loads predict risk of cervical cancer. Gynecol Oncol 2006; 102(1): 54-60.

[52] Muller M, Jacob Y, Jones L, et al. Large scale genotype comparison of human papillomavirus E2-host interaction networks provides new insights for e2 molecular functions. PLoS Pathog 2012; 8(6): e1002761.

[53] Lomalisa P, Smith T, Guidozzi F. Human immunodeficiency virus infection and invasive cervical cancer in South Africa. Gynecol Oncol 2000; 77(3): 460-3

[54] Moodley M, Moodley J, Kleinschmidt I. Invasive cervical cancer and human immunodeficiency virus (HIV) infection: a South African perspective. Int J Gynecol Cancer 2001; 11(3): 194-7.

[55] Stanley MFL, Davy VB, Matthew FC, et al. Association of HIV infection with distribution and viral load of HPV types in Kenya: a survey with 820 female sex workers. BMC Infect Dis 2010; 10: 18 .

[56] Didelot-Rousseau MN, Nagot N, Costes-Martineau V, et al. Human papillomavirus genotype distribution and cervical squamous intraepithelial lesions among high-risk women with and without HIV-1 infection in Burkina Faso. Br J Cancer 2006; 95(3): 355-62.

[57] Leroy V, Ladner J, De Clercq A, et al. Cervical dysplasia and HIV type 1 infection in African pregnant women: a cross sectional study, Kigali, Rwanda. The Pregnancy and HIV Study Group (EGE). Sex Transm Infect 1999; 75(2): 103-6.

[58] Moodley JR, Hoffman M, Carrara H, et al. HIV and pre-neoplastic and neoplastic lesions of the cervix in South Africa: a case-control study. BMC Cancer 2006; 6: 135.

[59] Sasco AJ, Jaquet A, Boidin E, et al. The challenge of AIDS-related malignancies in sub-Saharan Africa. PLoS One 2010; 5(1): e8621.

[60] Rezza G, Giuliani M, Branca M, et al. Determinants of squamous intraepithelial lesions (SIL) on Pap smear: the role of HPV infection and of HIV-1-induced immunosuppression. DIANAIDS Collaborative Study Group. Eur J Epidemiol 1997; 13(8): 937-43.

[61] Yamada R, Sasagawa T, Kirumbi LW, et al. Human papillomavirus infection and cervical abnormalities in Nairobi, Kenya, an area with a high prevalence of human immunodeficiency virus infection. J Med Virol 2008; 80(5): 847-55.

[62] Tornesello ML, Duraturo ML, Giorgi-Rossi P, et al. Human papillomavirus (HPV) genotypes and HPV16 variants in human immunodeficiency virus-positive Italian women. J Gen Virol 2008; 89(Pt 6): 1380-9.

[63] Tjalma WA, Depuydt CE. Don't forget HPV-45 in cervical cancer screening. Am J Clin Pathol 2012; 137(1): 161-2. 